\title{
Special issue on user-centred design and evaluation of ubiquitous groupware
}

\author{
Jacques Terken $\cdot$ Sriram Subramanian • \\ Massimo Zancanaro
}

Published online: 1 May 2007

(C) Springer-Verlag London Limited 2007

In the 1970s and 1980s, most work on supporting group and teamwork by means of computer technology focused on supporting decision processes for groups of people being physically together. With the advent of network technology, the focus shifted towards supporting group and teamwork at a distance and indeed for many people CSCW has become synonymous with supporting distributed collaboration, both synchronous and a-synchronous. Recently, with the advent of perceptual technologies (mainly computer vision, speech recognition), research projects have emerged that again focus on co-located collaboration. Today, the ambition is to develop systems that are able to perceive what is going on and to provide meaningful services to the group or team. The European Commission sponsors two large projects in this area: computers in the human interaction loop (CHIL, see http://chil.server.de) and augmented multi-party interaction (AMI, see http:// www.amiproject.org/). In the US, DARPA sponsors the project Cognitive Agent that Learns and Organizes (CALO, http://www.cse.ogi.edu/CHCC/Projects/CALO/ main.html). Canada has its Network for Effective Collab-

J. Terken $(\bowtie)$

Faculteit Industrial Design, Technische Universiteit

Eindhoven, Eindhoven, The Netherlands

e-mail: j.m.b.terken@tue.nl

S. Subramanian

Department of Computer Science,

University of Saskatchewan, Saskatoon, Canada

e-mail: sriram@cs.usask.ca

M. Zancanaro

Centro per la ricerca scientifica e tecnologica, Instituto Trentino di Cultura (ITC-irst), Povo, Italy

e-mail: zancana@itc.it oration Technologies through Advanced Research (NECTAR, see http://www.nectar-research.net), which among other things focuses on the use of perceptual technologies to support collaboration.

These projects have a strong technology focus and aim primarily at developing the perceptual and cognitive technologies and building demonstrators exemplifying the range of services that become possible through these technologies. However, from a design point of view, a strong technology focus raises two important issues. In the first place, in order to guide technology development so that our efforts will in the end provide services that are in fact useful and meaningful to society, we need to take a human-centred approach. In the second place, once we have service prototypes available, we need to assess the alleged benefits that those services provide.

For instance, the CHIL project has chosen meetings and lectures (or rather seminars) as its application domain and aims to provide services that help people to increase the productivity of their meetings or seminars. Questions are then raised as to what services would be useful in those contexts and how we can measure the productivity of people in meetings and seminars and the acceptance of these services. On the one hand, we feel that people have much experience with meetings and seminars, and have tuned the existing tools (such as pen and paper) and communicative strategies to optimize their productivity in such contexts. From this perspective, one should be extremely cautious to introduce computer technology in these contexts, since interacting with the services and applications may easily monopolize attention and distract people from their primary activity, and may therefore interfere with people's existing work practices instead of enhancing them. On the other hand, there is the widespread 
feeling that meetings are not very efficient and that there is much to improve about the way meetings are organized and conducted. For instance, people complain that much meeting time is spent on irrelevant details and that dominant participants have a tendency to monopolize the discussion. From this perspective, there seem to be opportunities for introducing technology to support people and improve their meeting effectiveness. However, also in the latter case we should be cautious to introduce technology and tune the services and applications to the needs of people rather than developing services just because the enabling technology is becoming available, because things only get worse if people are lured into interacting with the technology instead of focusing on their primary activity.

In order to discuss those issues, we organized a workshop on the topic of user-centred design and evaluation of services for human-human communication and collaboration, in conjunction with the International Conference on Multimodal Interaction, October 2005 in Trento, Italy. The aim was to bring together people from the projects mentioned above and other researchers interested to share experiences in the area of user-centred design and evaluation for co-located collaboration. Although in the area of CSCW user-centred design and evaluation have long traditions, we felt that the area of co-located (face-to-face) collaboration was sufficiently distinct to raise specific problems. In particular, the perceptual technologies enable the system to perceive what is going on, to reason about the course of events and to act proactively, which draws the design and research activities into the area of ubiquitous computing. And this brings aspects such as privacy, control and trust into the play, which need to be taken into consideration in the design and evaluation activities.

The workshop included a number of papers that we consider interesting for the wider scientific community, so we invited the workshop participants to rework their contribution and submit it for publication in this special issue. In addition, an open call was published inviting additional submissions. We coined the keyword ubiquitous groupware to replace the lengthy expression services for humanhuman communication and collaboration and to establish a relation between the focus on services supporting groups rather than individuals and on the ubiquitous and intelligent nature of those services. The papers selected for this special issue cover a range of topics in the field of user-centred design and evaluation of groupware for co-located collaboration. The first three papers directly relate to the design and evaluation of ubiquitous groupware. Pianesi and colleagues discuss the design of a service that provides feedback to people about their social behaviour in a meeting and thus may help them to adjust their behaviour and make future meetings more effective. Whittaker and colleagues describe the results of a user-centred approach to the evaluation and design of meeting browsers. Post and colleagues present the development of an evaluation method for co-located collaboration based on the input process outcome (IPO) model of group and teamwork. A further group of three papers relate more to the groupware aspect, while the ubiquitous aspect is less prominent. Still, we consider these papers relevant for the current special issue as they go into issues in the area of user-centred design of groupware for co-located collaboration. Pinelle and Gutwin present an adaptation of their collaboration usability analysis framework for the heuristic evaluation of tabletop groupware. Tan and colleagues present a method for conducting experiments to investigate the interaction with and communication about shared visual tools, and discuss evaluation metrics. Finally, Bailey and Biehl share their experiences concerning the adaptation of the wellknown paper prototyping technique for exploring the design space for collaboration in multi-display environments, and cast their experiences in a set of recommendations.

We believe that the papers in this special issue provide a useful overview of approaches towards the user-centred design and evaluation of groupware for co-located collaboration and expand the space of ubiquitous groupware by stimulating further research on this topic.

We thank the following people for reviewing papers, assisting us in selecting and improving papers for inclusion in the special issue: D. Aliakseyeu, A. Crabtree, M. Bevan, J. Di Micco, S. Fussell, J. Grudin, E. Hofer, J. Olson, M. Pallot, W. Prinz, D. Randall, H. Schaffers. 